\title{
Optimal Energy Dispatch in a Smart Micro-grid System using Economic Model Predictive Control
}

\author{
Mohamadou Nassourou ${ }^{1,2}$, Joaquim Blesa ${ }^{1,2}$, Vicenç Puig ${ }^{1,2}$ \\ ${ }^{1}$ Research Center for Supervision, Safety and Automatic Control (CS2AC), Universitat Politècnica de Catalunya (UPC), \\ Terrassa Campus, Gaia Building, Rambla Sant Nebridi 22, 08222 Terrassa,Barcelona. (Spain). \\ ${ }^{2}$ Institut de Robòtica i Informàtica Industrial (CSIC-UPC), \\ Carrer Llorens Artigas, 4-6, 08028 Barcelona (Spain)
}

\begin{abstract}
The problem of energy dispatch in heterogeneous complex systems such as smart grids cannot be efficiently addressed using classical control or ad-hoc methods. This paper discusses the application of Economic Model Predictive Control (EMPC) to the management of a smart micro-grid system connected to an electrical power grid. The considered system is composed of several subsystems, namely some photovoltaic (PV) panels, a wind generator, a hydroelectric generator, a diesel generator, and some storage devices (batteries). The batteries are charged with the energy from the PV panels, wind and hydroelectric generators, and they are discharged whenever the generators produce less energy than needed. The subsystems are interconnected via a DC Bus, from which load demands are satisfied. Modeling smart grids components is based on the generalized flow-based networked systems paradigm, and assuming energy generators to be stable, load demands and energy prices are known. This study shows that EMPC is economically superior to a two-layer hierarchical MPC.
\end{abstract}

Keywords: Smart grid, Energy Dispatch, Model Predictive Control, Economic Model Predictive Control

\section{Introduction}

Nowadays, electrical energy has a direct impact on people’s quality of life. The sector faces important challenges: growing energy needs of users, compelling environmental awareness and demanding economic environment ${ }^{1,2}$. Some of these challenges can be overcome with the help of smart grid systems. Smart grids are classical electrical grids that possess additionally some advanced modern components such as sensor systems, actuators, communication infrastructure, and a control system. They may be considered as examples of generalized flow-based networked systems ${ }^{3}$, as well as examples of Internet of Things.

Complex systems such as smart grids are MIMO (Multiple Input Multiple Output) systems incorporating multi-objective functions, whereby several heterogeneous components attempt to interact with each other requiring definitely a control strategy in order to optimize its efficiency and avoiding conflicting interactions.

Among the components that form smart grids nowadays, there are renewable or intermittent energy sources (e.g. solar, wind, hydroelectric generators), which require some predictive measures or actions in order to guarantee the reliability and stability of the energy supply. Furthermore, the complexity of multi-faceted load demands e.g. endogenous and exogenous demands increases the complexity of managing smart grids in an efficient and reliable manner. 
Classical control consisting basically of traditional feedforward and feedback PID controllers ${ }^{4,5}$ has been generally used to handle SISO (Single Input Single Output) systems usually described with transfer functions and frequency response. However, the control problem becomes quite difficult in the case of complex systems (as e.g. smart grids) because of the presence of multiple interacting variables and objective functions. Some of the drawbacks of classical control are its inability to handle in systematic way MIMO systems. Furthermore, classical control cannot handle constraints on process variables. To tackle these limitations, optimization ${ }^{6}$ based control was proposed in the 1960s. For instance, LinearQuadratic-Regulator (LQR) controllers solve optimization problems within a fixed window ${ }^{7}$. PID and LQR controllers do not possess the predictive ability. However, the LQG control scheme was the precursor to the development of models that are used in the Model Predictive Control (MPC) strategies. To some extent, MPC can be considered as a modified version of LQR formulation, which can deal with the operating constraints in a systematic manner.

MPC is a multivariable control strategy that uses ideally an accurate state space model, some possible constraints on the process variables, and an objective function to solve optimization problems $\mathrm{s}^{8,9}$. The predictive control solves an optimization problem using a moving time horizon window. MPC is not only able to predict in advance the next control, but it can also select the optimal control actions.

The development of MPC strategies to control hybrid energy systems such as smart grids has been carried out in several studies $^{10,11,12}$. MPC tracking requires some reference set-points, and its objective function is usually of quadratic form. It penalizes deviations of the states and control inputs from their reference trajectories while explicitly enforcing the constraints. However, the generation of reachable reference values at each step of the control horizon is not a trivial task, due to possible disturbances, set-point variations, time-dependent parameters and model inconsistencies, to mention just a few. This is actually one of the main drawbacks of MPC tracking. As a solution to this problem, real time optimizers (RTO) or steady-state target optimizers (SSTO) for pre-computing the reference values are usually introduced at an upper layer in the control strategies ${ }^{13,14}$. The pre-computed reference set-points are then forwarded to a lower-layer consisting of tracking MPC controllers acting as regulatory controllers for driving the system to desired operating points.

Even in the presence of an RTO, the problem of reachable trajectories might still occur due unexpected disturbances, setpoints changes and so on. In fact, there is a delay between the operations of the hierarchical layers, since the lower-layer should first receive the computed reference set-points from the upper-layer before starting executing its tasks. The need of an RTO may be avoided if the MPC strategy optimizes directly the parameters or variables of interest, thereby eliminating the requirement of reachable reference trajectories.

A standard Economic MPC (EMPC) strategy does not require reference trajectories ${ }^{15}$. Hence, it may be used in form of a single-layer approach to manage smart grid systems. Another approach to tackle the drawbacks of the traditional hierarchical scheme relies on the integration of an EMPC and MPC tracking in a hierarchical two-layer approach ${ }^{16}$. In this case, the upper layer consisting of an Economic MPC controller acts as a supervisory controller, while the lower-layer comprising some MPC tracking controllers performs the role of regulatory control. But this approach is not a solution to the delay problem that might occur in the coordination of the two-layers. $\operatorname{In}^{15,16}$, it has been shown that average asymptotic performance and/or closed-loop stability of EMPC can be guaranteed.

This paper proposes the application of EMPC to smart grids consisting of several heterogeneous energy sources. In this study, the standard EMPC strategy is applied to the problem of energy dispatch in a smart micro-grid. In order to appropriately assess the performance of the standard EMPC in tackling the problem at hand, a comparison with standard tracking MPC and the integration of both in a hierarchical two-layer approach has also been performed. A case study is used for illustration purposes based on a solar subsystem, a wind subsystem, a hydroelectric subsystem, a diesel generator subsystem, and some storage devices are integrated through a DC Bus into a power grid, for providing electrical energy to some DC-loads as well as to some residential and industrial areas. The DC Bus collects the energy generated by the subsystems and delivers it to the loads, and if necessary to the storage devices. The power delivered by all the subsystems must satisfy the load demand. The main issue to be resolved is the scheduling of the energy sources so that the costs are minimized. Renewable energies are influenced by weather conditions, economic situations and environmental issues. Practically, solar and wind energy systems require storage elements (e.g. batteries), while hydroelectric systems usually do not. Consequently, simultaneous control and coordination of the three energy systems is not a trivial task. Furthermore, the use of the diesel generator must be minimized because it is costly and contaminates the environment. Generally speaking, power generated by PV (photovoltaic) panels, wind turbines, and hydroelectric subsystems is less expensive than that from storage devices (batteries) and diesel generators. Solar and wind energies are more universal than hydroelectric energy. Additionally, solar energy is easier and cheaper to obtain than wind energy. In fact, sufficient wind power to rotate the 
turbines is principally available at some altitude, which is not the case with solar energy. Concerning the hydroelectric power, it requires the availability of water fall, but it is sometimes very cheap as soon as the power plant is built. It should not be noted that, during dry seasons hydroelectric power plants might not be able to produce the desired amount of energy.

The contributions of this paper are manifold:

a) Development of a control-oriented model of smart grids through mapping with generalized flow-based networked systems.

b) Development and application of MPC based energy management strategies for tackling the energy dispatch problem in a smart micro-grid consisting of several generators and storage elements.

c) Proving the standard EMPC superiority over its integration in single and two-layer schemes.

The paper is organized as follows: Section 2 describes a control-oriented model for smart grids. Section 3 formulates the MPC based strategies to be compared for the management of the smart grid under consideration. Section 4 describes an application example and presents the results of applying the three MPC based strategies. Concluding remarks and scope of the future work are presented in Section 5.

\section{Notation}

$\mathbb{R}, \mathbb{R}^{\mathrm{n}}, \mathbb{R}^{\mathrm{m} \times \mathrm{n}}$ represent the field of real numbers, the set of column real vectors of length $n$ and the set of $m$ by $n$ real matrices, respectively.

Scalars are denoted with lower case letters (e.g., a,b, $\beta, \ldots$ ), bold lower case letters (e.g., $\mathbf{x}, \mathbf{u}, \ldots$ ) represent vectors, and bold upper case letters (e.g., A, B,...) stand for matrices.

$\tilde{\mathbf{u}}(k)=\left(\mathbf{u}(0 \mid k), \ldots, \mathbf{u}\left(\mathrm{H}_{\mathrm{p}}-1 \mid k\right)\right) \in \mathbb{R}^{\mathrm{n}_{\mathrm{u}} \times \mathrm{H}_{\mathrm{p}}}$ stands for an ordered collection of $\mathrm{H}_{\mathrm{p}}$ predicted vectors $\mathbf{u}(k+i \mid k) \in \mathbb{R}^{\mathrm{n}_{\mathrm{u}}}$.

The operators $<, \leq,=,>$, $\geq$ should be understood as element-wise relations when applied to vectors.

\section{Control-oriented modeling}

\subsection{Modeling components of smart grid}

Electrical smart grids are instances of generalized flow-based networks, which basically consist of an interconnection of several components such as flow sources, links, nodes, storage, flow handling, and sink elements. Using such modelling paradigm $^{3,17,18}$, a generic electrical smart grid may be described using graph theory. In this work, a smart micro-grid comprising $n_{s}$ energy sources, $n_{x}$ storage elements, $n_{q}$ connection buses and $n_{d}$ loads is considered. The mathematical equation of each component is described in the following subsections.

\subsubsection{Flow source elements}

Energy generators and storage elements are considered as the flow sources, which deliver energy to the loads. Flow sources should be constrained since their productions are limited as follows

$$
P^{\min } \leq P(k) \leq P^{\max }
$$

where $P(k)$ is the supplied energy at time $k, P^{\min }$ is the lower limit (normally set to zero), and $P^{\max }$ is the upper limit determined by the installed power or by the net energy capacity constraint.

Some flow source elements such as renewable power sources have an additional time-dependent upper power limit determined by the availability of the resource (as e.g. wind velocity or solar irradiation) at instant $k$. Then, (1) can be rewritten as:

$$
P^{\min } \leq P(k) \leq P^{+}(k) \leq P^{\max }
$$

where $P^{+}(k)$ is the maximum energy generation profile at instant $k$.

\subsubsection{Link elements}

Power sources are connected to the network by means of flow control units termed as flow handling elements (active links) that accommodate the nature of power sources to the network connection requirements. These units can change direct current (DC) power or alternating current (AC) power to AC or DC for instance by means of inverters or rectifiers. They can also adapt the power sources voltages' values to fit in the connection network voltage standards using converters. Flow control units also select the supplied energy produced by its associated power source between the limits defined by 


$$
P^{\min } \leq P(k) \leq P^{\max }
$$

The output of the power source control unit $u_{\text {out }}(k)$ is given by the power supplied by the flow source multiplied by an electrical performance index $\eta$ :

$$
u_{\text {out }}(k)=\eta P(k)
$$

Then, considering (3) in the power limits defined by (1) or (2) we can obtain:

$$
\eta P^{\min } \leq u_{\text {out }}(k) \leq \eta P^{\max } \quad \text { or } \quad \eta P^{\min } \leq u_{\text {out }}(k) \leq \eta P^{+}(k) \leq \eta P^{\max }
$$

A flow handling element which neither increases nor decreases the incoming energy is referred as passive link. They are basically components that have the role of forwarding the incoming energy to other components of the grid. They could be as simple as switches or short transmission lines in some cases.

\subsubsection{Sink elements}

Sink elements represent the load demands, because they consume the flowing energy. Load demands denoted $d(k)$ (at time $k$ ) are bounded by the network connection energy capacity or by the maximum demands as established by users' contracts

$$
d^{\min } \leq d(k) \leq d^{\max }
$$

where $d^{\min }$ and $d^{\max }$ are the lower and upper bounds of the demands.

\subsubsection{Storage elements}

Batteries represent the storage elements ${ }^{19}$. A simplified dynamic behavior (i.e. charging and discharging) of this element can be described with the following equation ${ }^{20}$ :

$$
\operatorname{SOC}(k+1)=\operatorname{SOC}(k)+\eta_{c} P_{\text {in }}(k)-\eta_{d} P_{\text {out }}(k)
$$

where SOC denotes the battery State Of Charge, $\eta_{c}$ and $\eta_{d}$ are the charging efficiency and discharging efficiency of a given battery respectively, and $P_{\text {in }}(k)$ and $P_{\text {out }}(k)$ are the charged and discharged powers.

The storage capacity of the batteries is constrained as follows:

$$
S O C^{\min } \leq S O C(k) \leq S O C^{\max }
$$

with $S O C^{\min }$ and $S O C^{\max }$ the upper and lower limits of the stored energy.

\subsubsection{Node elements}

Node elements interconnect electrical grids' subsystems and loads. They are usually depicted with DC-buses, and are also known as junctions, for either merging or propagating energy flows to other smart grids' elements ${ }^{18}$. For any given node, it can be written:

$$
\sum_{i} P_{\text {in, } i}(k)=\sum_{j} P_{\text {out }, j}(k)
$$

where $P_{\text {out }, i}(k)$ and $P_{\text {out }, j}(k)$ correspond to the $i$-th node inflow and the $j$-th node outflow, respectively.

\subsection{Control-oriented model}

Nowadays, the state space representation is the standard manner of representing a model for implementing MPC strategies ${ }^{8,9}$.

Considering all the modeling components of a smart grid described in previous section, a typical smart grid can be described in state-space form using the following model 


$$
\begin{aligned}
& \mathbf{x}(k+1)=\mathbf{A} \mathbf{x}(k)+\mathbf{B} \mathbf{u}(k)+\mathbf{B}_{d} \mathbf{d}(k) \\
& \mathbf{E}_{u} \mathbf{u}(k)+\mathbf{E}_{d} \mathbf{d}(k)=0
\end{aligned}
$$

subject to the following constraints

$$
\begin{gathered}
u_{i}^{\min }(k) \leq u_{i}(k) \leq u_{i}^{\max }(k), \quad \forall k, \quad \forall i=1, \ldots, n_{u} \\
x_{j}^{\min } \leq x_{j}(k) \leq x_{j}^{\max }, \quad \forall k, \quad \forall j=1, \ldots, n_{x}
\end{gathered}
$$

where:

- $\quad \mathbf{x} \in \mathbb{R}^{\mathrm{n}_{\mathrm{x}}}$ is the state vector, where the states are the SOC of the storage elements (e.g. batteries). $x_{j}^{\min }$ and $x_{j}^{\max }$ are the charge lower and upper bounds of storage elements.

- $\mathbf{u} \in \mathbb{R}^{\mathrm{n}_{u}}$ is the control inputs vector which its components $u_{i}(k) \geq 0 \quad \forall i=1, \ldots, n_{u}$ i.e. $u_{i}^{\min }(k)=0$ in (11)

- $\quad \mathbf{d} \in \mathbb{R}^{\mathrm{n}_{\mathrm{d}}}$ denotes the disturbances vector (power demands of the consumers)

- The state matrices $\mathbf{A} \in \mathbb{R}^{\mathrm{n}_{\mathrm{x}} \times \mathrm{n}_{\mathrm{x}}} \quad \mathbf{B} \in \mathbb{R}^{\mathrm{n}_{\mathrm{x}} \times \mathrm{n}_{u}}, \mathbf{B}_{d} \in \mathbb{R}^{\mathrm{n}_{\mathrm{x}} \times \mathrm{n}_{d}}$ are system matrices that are obtained from the interconnections of the storage elements with the node and link elements.

- $\quad \mathbf{E}_{u} \in \mathbb{R}^{\mathrm{n}_{q} \times \mathrm{n}_{u}}$ and $\mathbf{E}_{d} \in \mathbb{R}^{\mathrm{n}_{q} \times \mathrm{n}_{d}}$ are matrices of suitable dimensions relating the supply and the load demand through the link and $n_{q}$ node elements (DC Bus(ses)).

\section{MPC strategies for the management of smart grids}

Once the micro-grid has been modelled, it is time to proceed to the MPC strategies for controlling the grid. As mentioned previously, we consider three MPC approaches to control the micro-grid. We start with the proposed EMPC approach, and then the MPC tracking is revisited, and finally we consider their integration in form of a hierarchical control scheme.

For the MPC formulations the following assumptions will be considered

Assumption 1. The states in $\mathbf{x}(k)$ and the demands in $\mathbf{d}(k)$ are assumed measured (or estimated) at time instant $k$, and the pair (A, B) is stabilizable.

Assumption 2. Flow source elements are stable (i.e. they are certain).

Assumption 3. Energy prices are known.

\subsection{Standard Economic MPC}

The main objectives of the EMPC strategy are the minimization of costs of production and distribution, as well as the guarantee of energy availability to satisfy load demands at any time in the smart grid system. To achieve these aims, it is necessary that the grid operates economically, safely, and smoothly. In fact, the same operational goals were used in ${ }^{3,21}$, where the problem of managing water distribution network of Barcelona was treated. In this study, we use similar operational goals for the management of electrical smart grids. EMPC is a proof of using classical MPC without specifying a reference trajectory. However, EMPC might introduce new theoretical challenges as mentioned in ${ }^{17}$.

\subsubsection{Power production and transportation cost}

Most of the economic costs associated with electrical power production are related to the purchase and maintenance of generators, as well as their accessories. Additionally, legal canons (taxes) and electricity costs can also be included in the associated economic costs.

The total cost is given by:

$$
f_{E}(k)=\left(\boldsymbol{\alpha}_{1}+\boldsymbol{\alpha}_{2}(k)\right)^{T} \mathbf{u}(k) \Delta t,
$$

where $\Delta t$ is the sampling time in seconds, $\boldsymbol{\alpha}_{1}$ is the time-independent vector related to economic costs and $\boldsymbol{\alpha}_{2}(k)$ is the timedependent vector of economic cost.

\subsubsection{Safety storage level}


This function is used to introduce a penalization of the control strategy that allows the SOC to go below the safety threshold $\boldsymbol{\delta}$ :

$$
\delta_{i}-\varepsilon_{i}(k) \leq x_{j}(k), \quad \forall k, \quad \forall j=1, \ldots, n_{x}
$$

where $\boldsymbol{\varepsilon}$ is a vector of slack variables that should be minimized by including an extra term in the MPC cost function

$$
f_{S}(k)=\boldsymbol{\varepsilon}(k)^{\mathrm{T}} \boldsymbol{\varepsilon}(k),
$$

$\boldsymbol{\varepsilon}(k)$ denotes the level of soft constraint violation., $\boldsymbol{\varepsilon}=0$ indicates that safety measures have been fully respected.

\subsubsection{Smoothness of the control action}

The rate of change of the control action ensures that consecutive control inputs increase or decrease in a smooth manner. This helps to reduce peaks of power in the DC Bus. The following quadratic term is used to penalize the control input's rate of change:

$$
f_{\Delta u}(k)=\Delta \mathbf{u}(k)^{\mathrm{T}} \Delta \mathbf{u}(k)
$$

where: $\Delta \mathbf{u}(k)$ is the control input variation defined as $\Delta \mathbf{u}(k)=\mathbf{u}(k)-\mathbf{u}(k-1)$.

\subsubsection{EMPC objective function}

Collecting all previous objectives, the EMPC cost function is defined as follows:

$$
J_{E M P C}(k)=\sum_{i=0}^{\mathrm{H}_{\mathrm{p}}-1}\left(\lambda_{1} f_{E}(k+i \mid k)+\lambda_{2} f_{S}(k+i \mid k)+\lambda_{3} f_{\Delta u}(k+i \mid k)\right)
$$

where index $k$ represents the current sampling time instant, index $i$ represents the time along the prediction and control horizons and $H_{p}$ is the prediction horizon. $\lambda_{1}, \lambda_{2}$, and $\lambda_{3}$ are weighting coefficients for prioritizing the objectives (13a), (13b) and (13c). They are positive scalar weights (dynamic or not) that can be used to control the effect of each operational goal on the overall performance of the system. In this work, these weights have tuned used the tuning procedure presented in 14 .

It might be important to remark that this EMPC objective function is actually a time varying function, since the first and second terms of the function (i.e. values of $\boldsymbol{\alpha}_{2}$ and $\boldsymbol{\varepsilon}$ ) are time dependent.

The EMPC optimization problem is formulated as follows:

$$
\begin{aligned}
& \min _{\tilde{\mathbf{u}}(k)} J_{E M P C}(k) \\
& \text { s.t. } \\
& \mathbf{x}(i+1 \mid k)=\mathbf{A x}(i \mid k)+\mathbf{B u}(i \mid k)+\mathbf{B}_{d} \mathbf{d}(i \mid k) \quad i=0, \ldots, \mathrm{H}_{\mathrm{p}}-1 \\
& \mathbf{E}_{u} \mathbf{u}(i \mid k)+\mathbf{E}_{d} \mathbf{d}(i \mid k)=0 \quad i=0, \ldots, \mathrm{H}_{\mathrm{p}}-1 \\
& \mathbf{x}(i \mid k) \geq \boldsymbol{\delta}-\boldsymbol{\varepsilon} \quad i=1, \ldots, \mathrm{H}_{\mathrm{p}} \\
& \mathbf{u}(i \mid k) \in\left[\mathbf{u}_{\text {min }}(i \mid k), \mathbf{u}_{\max }(i \mid k)\right] \quad i=0, \ldots, \mathrm{H}_{\mathrm{p}}-1 \\
& \mathbf{x}(i \mid k) \in\left[\mathbf{x}_{\min }, \mathbf{x}_{\max }\right] \quad i=1, \ldots, \mathrm{H}_{\mathrm{p}} \\
& \mathbf{x}(0 \mid k)=\mathbf{x}(k)
\end{aligned}
$$

$\tilde{\mathbf{u}}(k)=\left(\mathbf{u}(0 \mid k), \ldots, \mathbf{u}\left(\mathrm{H}_{\mathrm{p}}-1 \mid k\right)\right)$ is the sequence of optimal control actions. Only the first control action $\mathbf{u}(0 \mid k)$ is applied and then the optimization is repeated applying the receding horizon principle and initializing the initial state with the new states after applying $\mathbf{u}(0 \mid k)$. 
It is important to point out that EMPC could lead to possible loss of feasibility, particularly if parameters of the objective function such as energy prices and priority weights change the optimal cycle or the target state to some unreachable values for the given prediction horizon. The loss of feasibility could be solved for instance by using soft constraints, enlarging the prediction horizon or by including pseudo reference set-points in the cost function ${ }^{3}$. Additionally, a terminal state constraint based on (maximally) controlled invariant sets $^{23}$ can also be used to achieve recursive feasibility and stability:

where: $X_{\mathrm{T}}$ is the invariant terminal set.

$$
\mathbf{x}\left(\mathrm{H}_{\mathrm{p}} \mid k\right) \in X_{\mathrm{T}}
$$

Finally, in case of demands presenting repetitive periodic patterns, the prediction horizon $\mathrm{H}_{\mathrm{p}}$ is taken equal to the period and an additional constraint is added in the optimization problem (15) to guarantee that at the end of the period, the SOC of the storage elements is equal that at the beginning of the period

$$
\mathbf{x}\left(\mathrm{H}_{\mathrm{p}} \mid k\right)=\mathbf{x}(0 \mid k)
$$

This periodic constraint guarantees the stability and the feasibility of the solution. This is proved in a recent result from the authors (Wang, 2018). Moreover, the obtained solution coincides with the optimal one provided by the planner of the two layer scheme (Wang, 2018).

\subsection{Standard Tracking MPC}

Standard MPC tracking works by tracking some pre-specified set-points that facilitate a gradual transition to the desired reference set-point.

The cost function of standard tracking MPC is mostly expressed with a Quadratic Program (QP) form. For this work the following QP is selected:

$$
\begin{aligned}
J_{M P C}(k) & =\sum_{i=0}^{H p-1}\left[\left(\mathbf{x}(i \mid k)-\mathbf{x}_{r e f}(i \mid k)\right)^{T} \mathbf{Q}\left(\mathbf{x}(i \mid k)-\mathbf{x}_{r e f}(i \mid k)\right)+\left(\mathbf{u}(i \mid k)-\mathbf{u}_{r e f}(i)\right)^{T} \mathbf{R}\left(\mathbf{u}(i \mid k)-\mathbf{u}_{r e f}(i \mid k)\right)\right] \\
& +\left(\mathbf{x}\left(H_{p} \mid k\right)-\mathbf{x}_{r e f}\left(H_{p} \mid k\right)\right)^{T} \mathbf{S}\left(\mathbf{x}(i \mid k)-\mathbf{x}_{r e f}\left(H_{p} \mid k\right)\right)
\end{aligned}
$$

where $\mathbf{Q}, \mathbf{R}$ and $\mathbf{S}$ are the weighting matrices for prioitizing the objectives. In this work, these weights have tuned used the tuning procedure presented in ${ }^{22}$.

The optimization problem is defined as in (15) by replacing (14) with (17). Additionally, the terminal state constraint (16) is included.

If the reference trajectories i.e. $\mathbf{x}_{\mathrm{ref}}$ and $\mathbf{u}_{\mathrm{ref}}$ are not a priori known, then ${ }^{9,24}$ have proposed some methods for specifying some reference trajectories. Moreover, it is also possible to devise some purpose based methods for generating the reference trajectories.

One of the main disadvantages of MPC tracking is its requirement of reachable reference trajectories, which are not a priori easy to generate. To palliate this problem, the standard EMPC, a two-layer hierarchical (Economic MPC and MPC tracking) and a single-layer EMPC with pseudo reference set-points approaches are viable alternatives.

\subsection{Hierarchical two-layer approach}

A two-layer scheme can be used to eliminate non-reachable reference trajectories or even prevent infeasibility. An upper layer consisting of an Economic MPC and a lower layer comprising MPC tracking controllers are integrated together.

The Economic MPC is used as a supervisory controller, which computes reference trajectories (set-points) for the lower layer consisting of standard MPC tracking controllers responsible for driving the subsystems to desired set-points accordingly. A similar approach has been discussed in ${ }^{3,25}$. 


\subsubsection{Upper Layer EMPC}

It is made up of the standard EMPC presented in Section 3.1. The optimization problem is given in (15).

\subsubsection{Lower Layer MPC Tracking}

The MPC tracking discussed in Section 3.2 forms this layer, and computed states and control inputs by the upper layer are utilized as the reference set-points.

The optimization problem is formulated as in (15) by replacing (14) with (16). The stability of the system was achieved with the help of a terminal state constraint (16)

Even though the two-layer approach is a good method of generating reachable reference trajectories for the tracking MPC, it introduces at the same time another problem namely a delay in the coordination of the two layers, and possibly infeasibility of the optimization problem in the presence of changing operating patterns.

\subsection{Single-layer Economic MPC with pseudo reference set-points}

The delay problem as well as the possible loss of feasibility could be solved by embedding a pseudo reference tracking component in the standard EMPC as discussed in $^{3}$. The economic optimisation (EMPC) and the tracking formulation (MPC tracking) are performed within a single layer. The MPC optimization problem is formulated as in Eq (17) by substituting $\mathbf{x}(i \mid k)$ with $\mathbf{x}^{r}(i \mid k)$, and $\mathbf{u}(i \mid k)$ with $\mathbf{u}^{r}(i \mid k)$ in $J_{E M P C}(k)$

$$
\begin{aligned}
& \min _{\mathbf{u}(k), \mathbf{u}^{r}(k)}\left(\gamma_{0} J_{E M P C}(k)+\gamma_{1} J_{M P C}(k)\right) \\
& \text { s.t. } \\
& \mathbf{x}(i+1 \mid k)=\mathbf{A x}(i \mid k)+\mathbf{B u}(i \mid k)+\mathbf{B}_{d} \mathbf{d}(i \mid k) \quad i=0, \ldots, \mathrm{H}_{\mathrm{p}}-1 \\
& \mathbf{E}_{u} \mathbf{u}(i \mid k)+\mathbf{E}_{d} \mathbf{d}(i \mid k)=0 \quad i=0, \ldots, \mathrm{H}_{\mathrm{p}}-1 \\
& \mathbf{x}(i \mid k) \geq \boldsymbol{\delta}-\boldsymbol{\varepsilon} \quad i=1, \ldots, \mathrm{H}_{\mathrm{p}} \\
& \mathbf{u}(i \mid k) \in\left[\mathbf{u}_{\min }(i \mid k), \mathbf{u}_{\max }(i \mid k)\right] \quad i=0, \ldots, \mathrm{H}_{\mathrm{p}}-1 \\
& \mathbf{x}(i \mid k) \in\left[\mathbf{x}_{\min }, \mathbf{x}_{\max }\right] \quad i=1, \ldots, \mathrm{H}_{\mathrm{p}} \\
& \mathbf{x}(0 \mid k)=\mathbf{x}(k) \\
& \mathbf{x}^{r}(i+1 \mid k)=\mathbf{A x}^{r}(i \mid k)+\mathbf{B u}^{r}(i \mid k)+\mathbf{B}_{d} \mathbf{d}(i \mid k) \quad i=0, \ldots, \mathrm{H}_{\mathrm{p}}-1 \\
& \mathbf{E}_{u} \mathbf{u}^{r}(i \mid k)+\mathbf{E}_{d} \mathbf{d}(i \mid k)=0 \quad i=0, \ldots, \mathrm{H}_{\mathrm{p}}-1 \\
& \mathbf{u}^{r}(i \mid k) \in\left[\mathbf{u}_{\text {min }}^{r}(i \mid k), \mathbf{u}^{r}{ }_{\text {max }}(i \mid k)\right] \quad i=0, \ldots, \mathrm{H}_{\mathrm{p}}-1 \\
& \mathbf{x}^{r}(i \mid k) \in\left[\mathbf{x}_{\text {min }}^{r}, \mathbf{x}_{\text {max }}^{r}\right] \quad i=1, \ldots, \mathrm{H}_{\mathrm{p}} \\
& \mathbf{x}^{r}(0 \mid k)=\mathbf{x}^{r}(k)
\end{aligned}
$$

where $\gamma_{0}$ and $\gamma_{1}$ are scalar weights introduced to make a trade-off between economic and tracking performance that should be tuned appropriately. In this work, these weights have tuned used the tuning procedure presented in ${ }^{22}$.

\section{Application Example}

\subsection{Description}

In this section, we present a micro-grid that comprises: two batteries, three loads, one external grid connection, one DC Bus, some photovoltaic panels, one wind generator, one hydroelectric generator and one diesel generator. The external grid connection is a sink when it buys energy and a source when it sells energy.

All the components (excluding loads) are considered to be manipulated inputs. The states of the micro-grid are defined to be state of charge of the storage elements.

The smart micro-grid is depicted in Figure 1. 


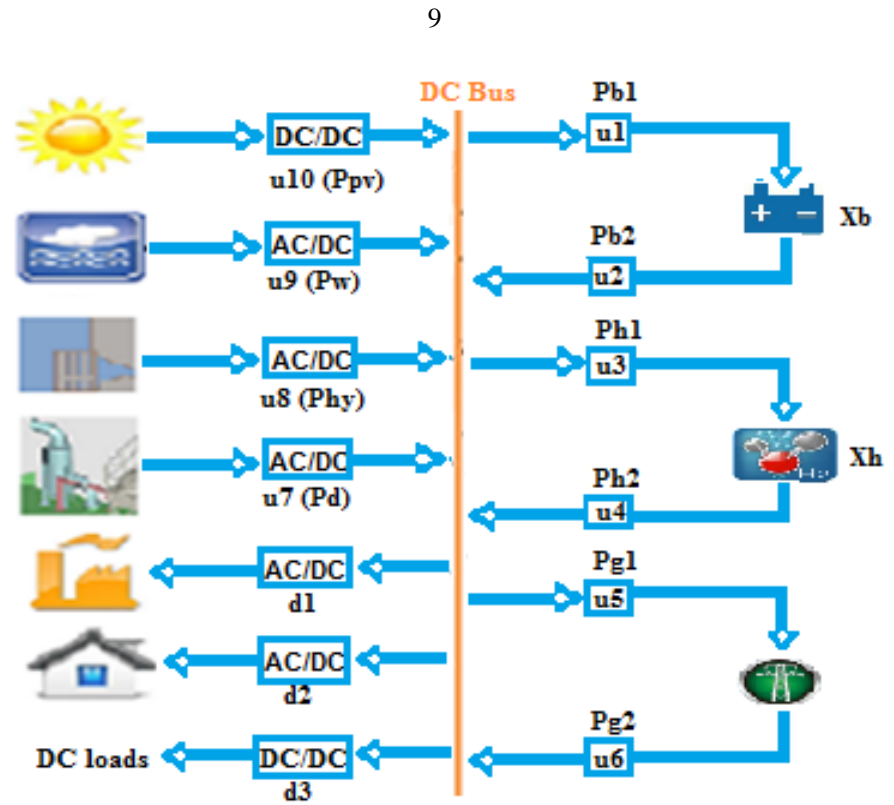

Figure 1. The smart micro-grid

\subsection{Control-oriented model}

\section{States:}

$x_{b}$ and $x_{h}$ stand for the SOC of the lead-acid and hydrogen batteries respectively, such that the state vector is defined as $\mathbf{x}(k) \triangleq\left(x_{b}(k), x_{h}(k)\right)^{\mathrm{T}}$

\section{Control inputs:}

$P_{b 1}$ and $P_{b 2}$ represent the lead-acid battery's charged and discharged powers respectively;

$P_{h 1}$ and $P_{h 2}$ represent the hydrogen battery's charged and discharged powers respectively;

$P_{g 1}$ and $P_{g 2}$ represent the exported and imported powers into/from the external grid;

$P_{d}, P_{h y}, P_{p v}$, and $P_{w}$ denote the power delivered to the DC Bus by the diesel, hydroelectric, wind, and photovoltaic generators respectively;

Thus, the control input is defined as follows

$$
\mathbf{u}(k) \stackrel{\wedge}{=}\left(P_{b 1}(k), P_{b 2}(k), P_{h 1}(k), P_{h 2}(k), P_{g 1}(k), P_{g 2}(k), P_{d}(k), P_{h y}(k), P_{w}(k), P_{p v}(k)\right)^{T}
$$

\section{Disturbance variables:}

$d_{1}$ is the industrial load, $d_{2}$ is the residential load, while $d_{3}$ is the DC-load. The load demand vector $\mathbf{d}$ consists of the three loads. $\mathbf{d}(k) \triangleq\left(d_{1}(k), d_{2}(k), d_{3}(k)\right)^{\mathrm{T}}$

The system matrices and vectors and its constraints are expressed as follows:

$$
\begin{aligned}
& \mathbf{A}=\left(\begin{array}{ll}
1 & 0 \\
0 & 1
\end{array}\right) \\
& \mathbf{B}=\left(\begin{array}{cccccccccc}
\eta_{1} \eta_{b c} & -\eta_{2} \eta_{b d} & 0 & 0 & 0 & 0 & 0 & 0 & 0 & 0 \\
0 & 0 & \eta_{3} \eta_{h c} & -\eta_{4} \eta_{h d} & 0 & 0 & 0 & 0 & 0 & 0
\end{array}\right) \\
& \mathbf{B}_{p}=\left(\begin{array}{lll}
0 & 0 & 0 \\
0 & 0 & 0
\end{array}\right)
\end{aligned}
$$

where: $\eta_{h c}$ and $\eta_{h d}$ are the charging efficiency and discharging efficiency of the hydrogen battery respectively; $\eta_{b c}$ and $\eta_{b d}$ are the charging efficiency and discharging efficiency of the lead-acid battery respectively.

The physical bounds of states and control inputs are defined as follows:

$\mathbf{x}^{\min }=\left(\begin{array}{ll}0 & 0\end{array}\right)^{\mathrm{T}}$,

$\mathbf{x}^{\max }=\left(\begin{array}{lll}100 & 100\end{array}\right)^{\mathrm{T}}$

$\mathbf{u}_{k}^{\min }=\left(\begin{array}{llllllllll}0 & 0 & 0 & 0 & 0 & 0 & 0 & 0 & 0 & 0\end{array}\right)^{\mathrm{T}}$,

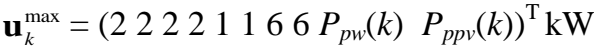


where $P_{p w}(k) \leq 18 \mathrm{Kw}$ and $P_{p p v}(k) \leq 18 \mathrm{~kW}$ are the energy generation profiles of the wind and photovoltaic generators respectively.

The energy generation and consumption profiles are created by a forecast module that is not discussed in this study.

The energy balance of the DC Bus according to (9b) is given by:

$\mathbf{E}_{\mathbf{u}}=\left(-\eta_{1} \eta_{2}-\eta_{3} \eta_{4}-\eta_{5} \eta_{6} \eta_{7} \eta_{8} \eta_{9} \eta_{10}\right), \mathbf{E}_{\mathbf{d}}=(-1-1-1)$

where $\eta_{i} \leq 1 \forall i=1, \ldots, 10$ is the electrical performance index of associated control units, and $\eta_{\mathrm{i}}=1$.

$\boldsymbol{\alpha}^{1} \triangleq\left(\alpha_{1}^{p b 1}, \alpha_{1}^{p b 2}, \alpha_{1}^{h 1}, \alpha_{1}^{h 2}, \alpha_{1}^{g 1}, \alpha_{1}^{g 2}, \alpha_{1}^{d}, \alpha_{1}^{h y}, \alpha_{1}^{w}, \alpha_{1}^{p v}\right)^{T}$

where: $\alpha_{1}^{p b 1}, \alpha_{1}^{p b 2}, \alpha_{1}^{h 1}, \alpha_{1}^{h 2}, \alpha_{1}^{g 1}, \alpha_{1}^{g 2}, \alpha_{1}^{d}, \alpha_{1}^{h y}, \alpha_{1}^{w}, \alpha_{1}^{p v}$ are fixed costs corresponding to the control input variables respectively. Their values are shown in Table 1.

In this study energy prices are considered to be time independent, which implies that $\boldsymbol{\alpha}_{2}$ is constant, and for the sake of simplicity it is set to zero as shown in Table 1.

MPC objective function's matrices and parameters

$\mathbf{Q}=\left(\begin{array}{ll}1 & 0 \\ 0 & 1\end{array}\right)$

$\mathbf{R}=\operatorname{diag}\left(\mathrm{c}_{\mathrm{b}}, \mathrm{c}_{\mathrm{b}}, \mathrm{c}_{\mathrm{h}}, \mathrm{c}_{\mathrm{h}}, \mathrm{c}_{\mathrm{g}}, \mathrm{c}_{\mathrm{g}}, \mathrm{c}_{\mathrm{d}}, \mathrm{c}_{\mathrm{hy}}, \mathrm{C}_{\mathrm{w}}, \mathrm{c}_{\mathrm{pv}}\right)$

$\mathbf{S}_{\mathbf{x}}=\mathbf{W}_{\mathrm{c}} \cdot \mathbf{Q}$

where: $c_{b}, c_{h}, c_{g}, c_{d} c_{d}, c_{p v}, c_{w}$ and $c_{h y}$ are non-negative weight coefficients $(\leq 1)$ for the lead-acid battery, hydrogen battery, grid connection, diesel, solar, wind, and hydroelectric generators respectively. Their values are shown in Table 1 . $\mathbf{W}_{\mathrm{c}}$ is a scalar weighting factor for the terminal state.

We performed some simulations for some days where all the generators had to be used. Additionally the batteries, the external grid, and the diesel generator were operated whenever there was shortage of power to satisfy load demands. The simulations were carried out for four days. The diesel generator delivered between $1 \mathrm{kWh}$ and $1.3 \mathrm{kWh}$ in summer during the first six hours of the day and in winter in the afternoon. The batteries discharged $2 \mathrm{kWh}$ in the first hour and $1 \mathrm{kWh}$ in the second hour of the day. The external grid offered $1 \mathrm{kWh}$ during the second hour of the day. All MPC controllers in this study have been implement YALMIP in Matlab environment and the Gurobi solver.

\begin{tabular}{|c|c|c|c|}
\hline \multicolumn{2}{|c|}{ System parameters } & \multicolumn{2}{|c|}{ Control parameters } \\
\hline Parameters & Values in $\mathrm{kW}$ & Parameters & Values \\
\hline $\mathrm{P}_{\mathrm{pv}}^{\max }$ & 6.75 & $\mathrm{H}_{\mathrm{p}}$ & 24 \\
\hline $\mathrm{P}_{\mathrm{w}}^{\max }$ & 7.75 & $C_{p v}$ & 0.2 \\
\hline $\mathrm{P}_{\text {hy }}^{\max }$ & 4 & $C_{W}$ & 0.3 \\
\hline $\mathrm{P}_{\mathrm{d}}^{\max }$ & 6 & $C_{\text {hy }}$ & 0.4 \\
\hline $\mathrm{P}_{\mathrm{b} 1}^{\max }$ & 2 & $\mathrm{C}_{\mathrm{b}}$ & 0.75 \\
\hline $\mathrm{P}_{\mathrm{b} 2}^{\max }$ & 2 & $c_{h}$ & 0.75 \\
\hline $\mathrm{P}_{\mathrm{h} 1}^{\mathrm{max}}$ & 2 & $c_{d}$ & 1 \\
\hline $\mathrm{P}_{\mathrm{h} 2}^{\max }$ & 2 & $C_{g}$ & 0.75 \\
\hline $\mathrm{P}_{\mathrm{g} 1}^{\max }$ & 1 & $\mathbf{Q}$ & as defined previously \\
\hline $\mathrm{P}_{\mathrm{g} 2}^{\max }$ & 1 & $\mathbf{R}$ & as defined previously \\
\hline$\eta_{\mathrm{bc}}$ & 0.95 & $\boldsymbol{\alpha}_{2}$ & $\boldsymbol{\alpha}_{1}$ \\
\hline$\eta_{\text {bd }}$ & 1 & \multirow{4}{*}{\multicolumn{2}{|c|}{$\begin{array}{l}\alpha_{1}^{p b 1}=\alpha_{1}^{p b 2}=\alpha_{1}^{h 1}=\alpha_{1}^{h 2}=0.02, \alpha_{1}^{g 1}=\alpha_{1}^{g 2}=3.3, \\
\alpha_{1}^{d}=4.3, \alpha_{1}^{h y}=2.3, \alpha_{1}^{w}=1.6, \alpha_{1}^{p v}=1.4, \\
\lambda_{1}=2500, \lambda_{2}=12, \lambda_{3}=0.1\end{array}$}} \\
\hline$\eta_{\mathrm{hc}}$ & 0.85 & & \\
\hline$\eta_{\text {hd }}$ & 1.0 & & \\
\hline$\Delta$ & {$\left[\begin{array}{ll}35 & 35\end{array}\right]^{\mathrm{T}}$} & & \\
\hline
\end{tabular}

Table 1. System and control parameters' values 
The higher a coefficient is, the stronger is the penalization of its corresponding subsystem. The expert algorithm presented previously was used to select the value of each coefficient.

The expected data of energy flow from the generators is given for a single weekday. Figure 2 shows the forecasted energy production by the PV and wind generators, as well as load demands.
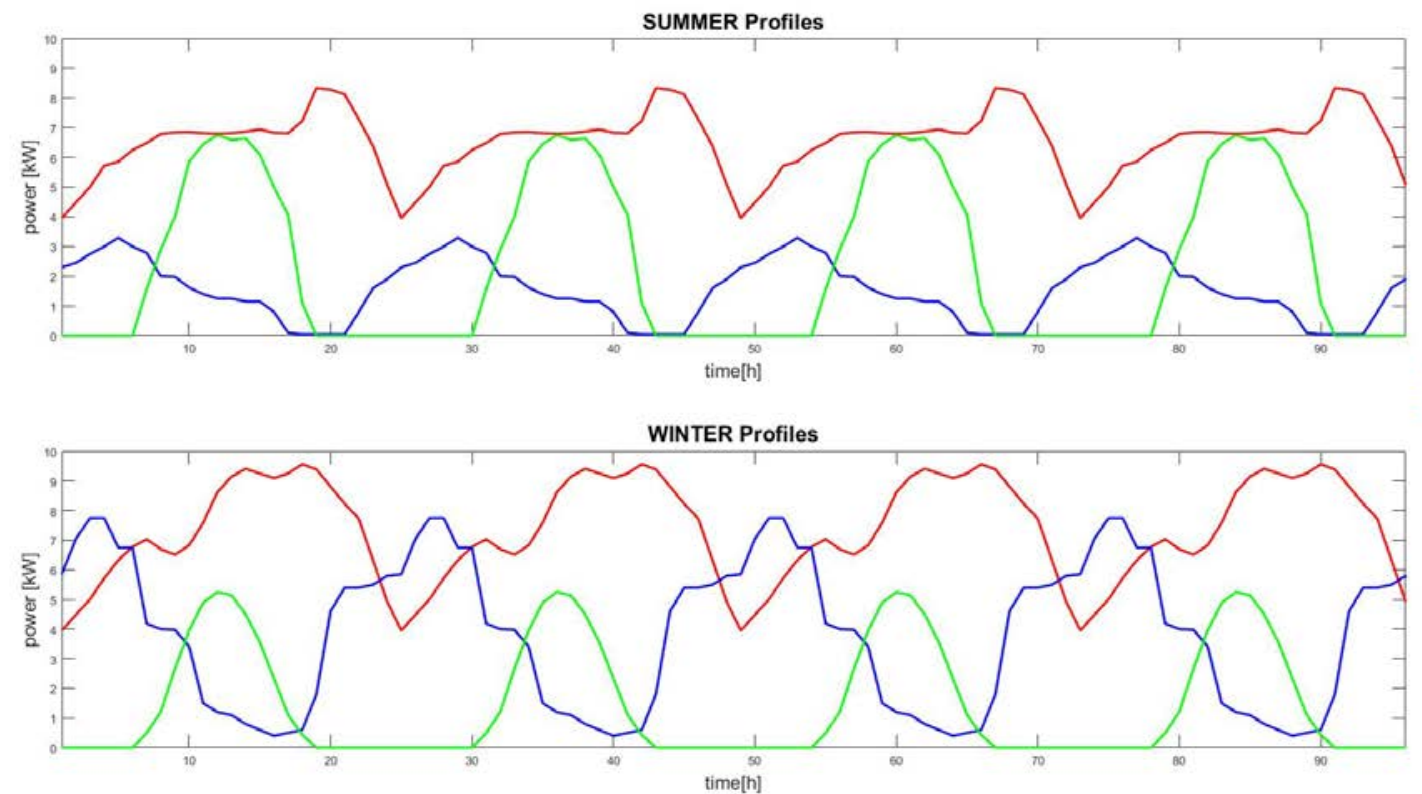

Fig. 2. Forecasted profiles of the generators and load demands

The profile of a generator represents the maximum power that can be ideally produced by the generator at time $k$. The following figures show some sample comparisons of the energy production in summer and winter scenarios

\section{A) Summer results}
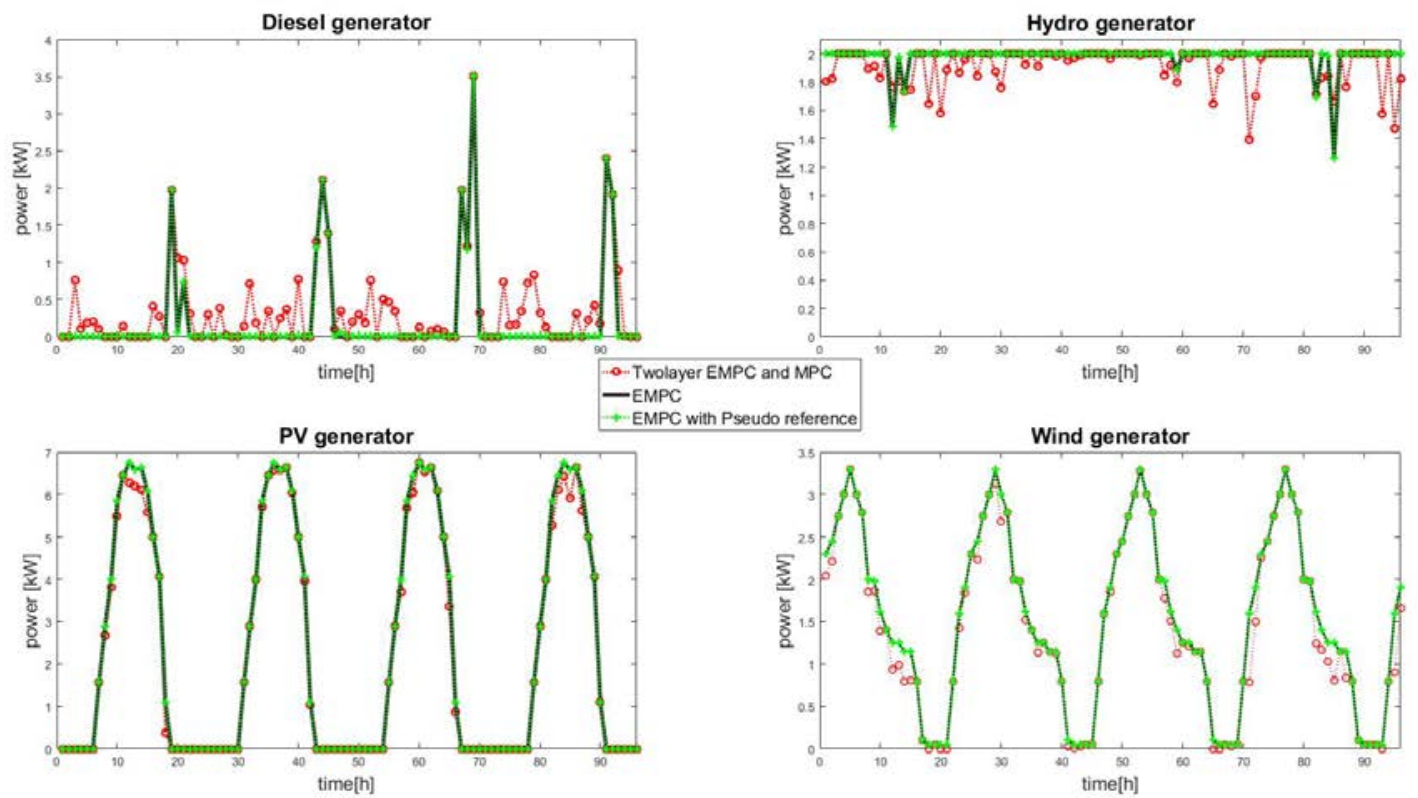

Figure 3. Plots of the energy generation in summer 


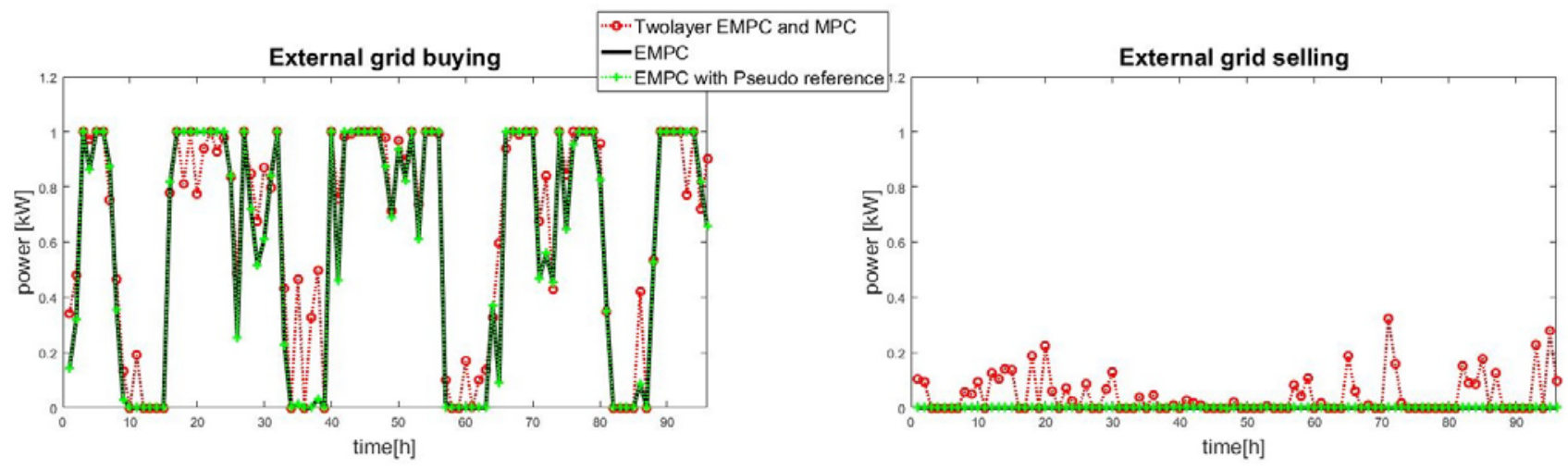

Figure 4. Plots of the utilization of the external grid in summer

\section{B) Winter results}
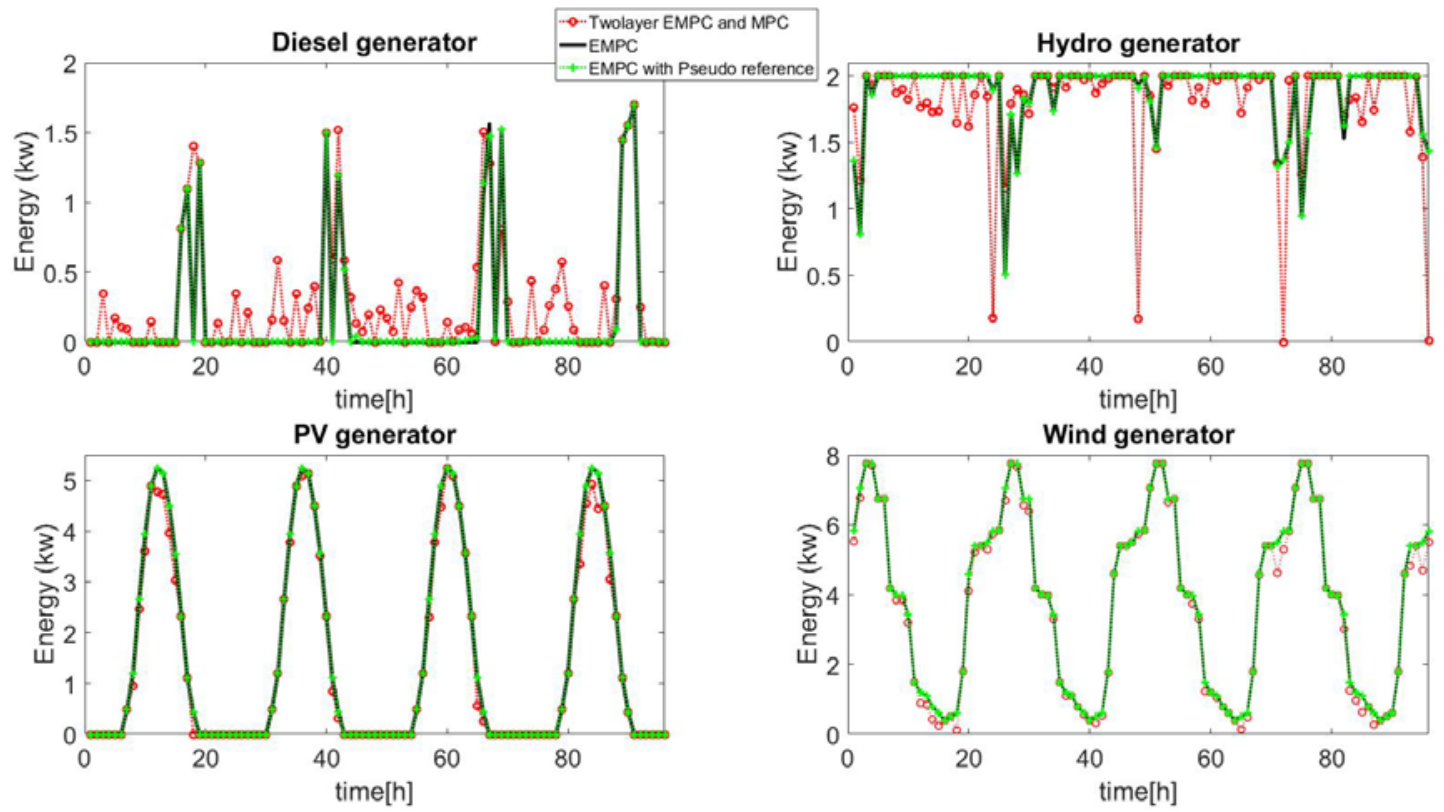

Figure 5. Plots of the energy generation in winter

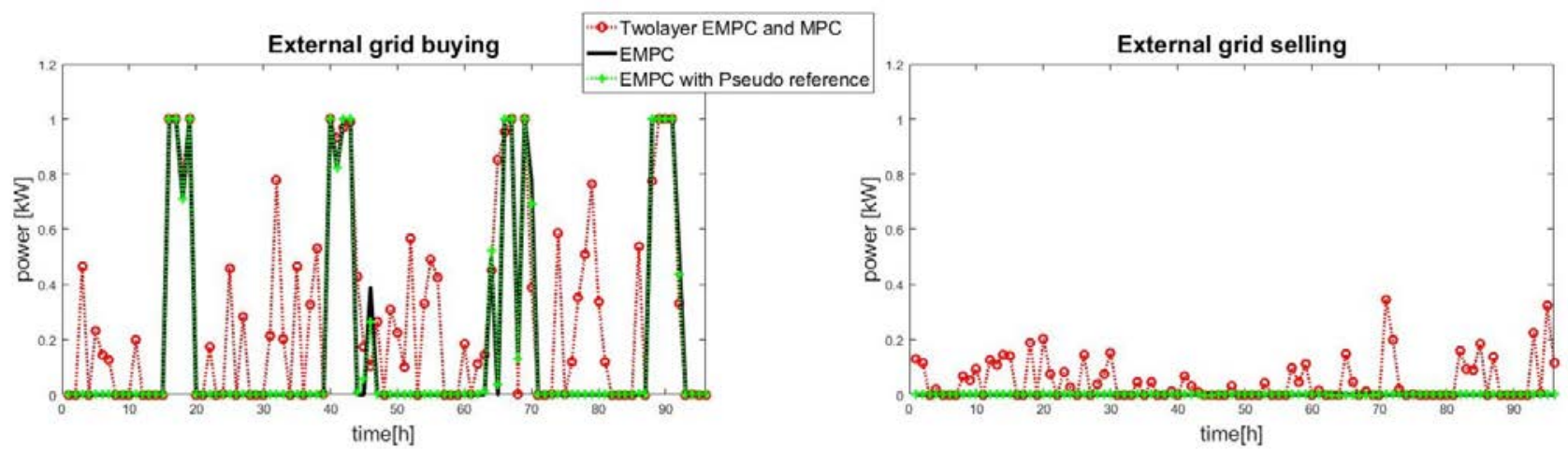

Figure 6. Plots of the utilization of the external grid in winter 
The goal of the proposed strategies is to reduce the energy generation costs. Energy from the diesel generator is more expensive than from renewable energy sources. As it can be seen from Figures 3 to 6, the EMPC strategy performs similarly to EMPC with pseudo-reference while they present better performance than the two-layer scheme since the reduction of energy production using the diesel generator and the maximization of the use of renewable energies (wind generator and solar panels). Moreover, two-layer scheme is more dependent on the external grid, because it presents more interaction with the external grid, in particular during the winter.

Table 2 displays the comparison of the three MPC approaches. The results presented in this table support the previous conclusions extracted from the figures. These results are in accordance with those presented in ${ }^{14}$ in the context of water distribution networks.

Regarding the computational cost, Table 3 shows that the EMPC is the less demanding strategy while the EMPC with pseudo-reference is the most demanding one.

\begin{tabular}{|l|c|c|c|}
\hline & Economic MPC & $\begin{array}{c}\text { EMPC + pseudo reference } \\
\text { (one-layer; } \gamma_{0}=1, \gamma_{1}=1 \text { ) }\end{array}$ & $\begin{array}{c}\text { EMPC + MPC tracking } \\
\text { (two-layer) }\end{array}$ \\
\hline Summer economic cost & 632.61 & 632.66 & 664.37 \\
\hline Winter economic cost & 667.50 & 667.63 & 695.93 \\
\hline
\end{tabular}

Table 2. Quantitative analysis of the economic costs

\begin{tabular}{|l|c|c|c|}
\hline & Economic MPC & $\begin{array}{c}\text { EMPC + pseudo reference } \\
\text { (one-layer; } \gamma_{0}=1, \gamma_{1}=1 \text { ) }\end{array}$ & $\begin{array}{c}\text { EMPC + MPC tracking } \\
\text { (two-layer) }\end{array}$ \\
\hline Seconds per iteration & $2.1 \times 10^{-2}$ & $6.2 \times 10^{-2}$ & $3.75 \times 10^{-2}$ \\
\hline
\end{tabular}

Table 3. Quantitative analysis of computational cost

\section{Conclusion}

In this work, we discussed the application of three variations of Model Predictive Control strategies for controlling a grid connected electrical micro-grid consisting of several subsystems namely some photovoltaic (PV) panels, a wind generator, a hydroelectric generator, a diesel generator, and some storage devices (batteries).

In particular, we have first considered the standard EMPC, EMPC with pseudo-reference and a hierarchical two-layer MPC consisting of the integration of a planner and a tracker that uses standard MPC.

Comparing the daily economic costs of the subsystems in the proposed case study, we have shown that standard EMPC and EMPC with pseudo-reference yields similar results, but at an increased computational cost for the latter. Both strategies are clearly economically superior to the hierarchical two-layer MPC.

The result of this study shows that, EMPC strategy can be successfully used to control energy dispatch in smart micro-grids. Future works for completing this study will be centered on considering uncertainties in the system as in demand and price energies forecast and green energy power availability that will imply the use of robust optimization methods.

\section{Acknowledgments}

This work has been partially funded by the Spanish Ministry of Economy and Competitiveness (MINECOP) and FEDER through the project HARCRICS (ref. DPI2014-58104- R) and through the grant IJCI-2014-20801 and by the Spanish State Research Agency through the María de Maeztu Seal of Excellence to IRI (MDM-2016-0656)

\section{References}

1. Wu X, Hu X, Moura S, Yin X, Pickert V. Stochastic control of smart home energy management with plug-in electric vehicle battery energy storage and photovoltaic array. Journal of Power Sources 2016; 333: pp 203-212 
2. Wu X, Hu X, Teng Y, Qian S and Cheng R. Optimal integration of a hybrid solar-battery power source into smart home nanogrid with plug-in electric vehicle. Journal of Power Sources 2017; 363: pp 277-283.

3. Grosso J M, On Model Predictive Control for Economic and Robust Operation of Generalized Flow-based Networks, PhD thesis, UPC Barcelona, Spain, 2014

4. Åström K J. Control System Design. 2002.

5. Åström K J. Advanced PID Control. With T Hägglund. ISA, 2005.

6. Nassourou M. Design of Digital Joint Controller for Dexterous Climbing Robot. Master.thesis, ISNM, University of Lübeck, Germany, 2005.

7. Murray R. M.Optimization-Based Control. DRAFT v1.4a, California Institute of Technology, 2008.

8. Liuping, W. Model Predictive Control System Design and Implementation Using MATLAB, ISBN 978-1-84882-330-3, 2009.

9. Maciejowski J M. Predictive Control with Constraints, Prentice Hall, New Jersey, USA, 2002.

10. Halvgaard R. Model Predictive Control for Smart Energy Systems. PhD thesis, DTU Denmark, 2014.

11. Prodan I E. A model predictive control framework for reliable microgrid energy management. Journal Elsevier Electrical Power and Energy Systems 2014; 61 (2014) 399-409.12. Pereira M, Limon D, Alamo T and Valverde L. Application of Periodic Economic MPC to a Grid-Connected Micro-grid. IFAC-PapersOnLine 2015; 48 (23): $513-518$.

13. Tatjewski P. Advanced control and on-line process optimization in multilayer structures. In: Annual Reviews in Control, 32(1), 2008.

14. Grosso J M, Ocampo-Martinez C, Puig V, Limon D, Pereira M. Economic MPC for the Management of Drinking Water Networks, 2014 European Control Conference (ECC), Strasbourg, 2014, pp. 790-795.

15. Rawlings J B, Angeli D Bates. Fundamentals of economic model predictive control. In: Proc. 51st IEEE Conference on Decision and Control (CDC), 2012; pages 3851-3861.

16. Ellis M, Christofides P D. Integrating dynamic economic optimization and model predictive control for optimal operation of nonlinear process systems. Control Engineering Practice, 2014; 22(0):242-251.

17. Acakpovi A, Ben Hagan E, Fifatin F. Review of Hydropower Plant Models. International Journal of Computer Applications, 2014; (0975 - 8887) Volume 108 - No 18.

18. Puig V, Costa R, Sampietro J.L. Researcher. Economic MPC for the Energy Management of Hybrid Vehicles including Fuel Cells and Supercapacitors, UK Control, Belfast, UK. 2016.

19. Hu X, Zou C, Zhang C and Li Y. Technological Developments in Batteries: A Survey of Principal Roles, Types, and Management Needs. IEEE Power and Energy Magazine 2017; 15(5): pp. 20-31.

20. Zhu B, Tazvinga H. and Xia, X. Switched Model Predictive Control for Energy Dispatching of a Photovoltaic-DieselBattery Hybrid Power System. IEEE Transactions on Control Systems Technology 2015; 23(3): pp. 1229-1236.

21. Ocampo-Martinez C, Puig V, Grosso J M, Montes de Oca S. Distributed MPC Made Easy, chapter 30: Multi-layer Decentralized Predictive Control of Large-Scale Networked Systems. Springer-Verlag, 2013.

22. Rodrigo Toro, Carlos Ocampo-Martínez, Filip Logist, Jan Van Impe, Vicenç Puig, Tuning of Predictive Controllers for Drinking Water Networked Systems, IFAC Proceedings Volumes 2011, 44 (1): 14507-14512.

23. F. Blanchini, Set invariance in control, Automatica, 1999, 35(11):1747-1767.

24. Rossiter J A. Model-Based Predictive Control: A Practical Approach. CRC Press, Boca Raton, FL, 2003.

25. Liu W Qi, J Chen,X, Christofides, P D. Supervisory Predictive Control of an Integrated Wind/Solar Energy Generation and Water Desalination System. In: Proceedings of the 9th International Symposium on Dynamics and Control of Process Systems, 2010; Leuven, Belgium.

26. Nassourou M, Puig V, Blesa J. Robust optimization based energy dispatch in smart grids considering demand uncertainty Journal of Physics, 2017;Conference Series, 783 (1), art. no. 012033.

27. Wang, Y., Muñoz de la Pela, D., Puig, V., Cembrano, G. A Novel Formulation of Economic Model Predictive Control for Periodic Operations. In Proceedings of the European Control Conference 2018. Limassol, Cyprus. 